\section{Curso de imersão em diabetes como técnica educativa para profissionais médicos}

\author{
Diabetes immersion training as teaching method to medical practitioners
}

Patrícia H. Zanoni ${ }^{1}$, Maria Cândida Ribeiro Parisi ${ }^{1}$,

Sharon N. Admoni' ${ }^{1}$ Márcia S. Queiroz', Marcia Nery

\section{RESUMO}

Este estudo avaliou a eficácia do método de ensino teórico e prático sobre diabetes e a atitude de profissionais médicos quanto à realização de controle glicêmico intensivo. Participaram de um curso de imersão em diabetes, com dois dias de duração, 48 médicos-residentes de clínica médica ou endocrinologia. Os participantes receberam treinamento de monitorização de glicemia capilar, técnicas de aplicação de insulina e contagem de carboidratos, sendo orientados a se portarem como diabéticos e a seguir prescrição médica individual. Foram avaliados através de questionários. No questionário de conhecimentos, observou-se um aumento significante de $12 \%$ no índice de acertos entre o início e o final do curso $(61,2 \%$ e $73,2 \%$, respectivamente, com $\mathrm{p}<0,0001)$. Antes do curso, $70,8 \%$ dos participantes diziam ter dificuldades na contagem de carboidratos e 89,6\%, na automonitorização glicêmica. Após a experiência prática, 82,9\% dos participantes encontraram dificuldades na realização de contagem de carboidratos e 80,8\%, na automonitorização; $40,4 \%$ fizeram uso de todas as medicações prescritas e $36,1 \%$ monitorizaram todas as glicemias. Os resultados deste estudo mostram que esse tipo de curso é eficaz para a aquisição de conhecimentos e contribui com a sensibilização do profissional médico quanto às dificuldades cotidianas enfrentadas pelo portador de diabetes melito na aderência às recomendações. Arq Bras Endocrinol Metab. 2009;53(3):355-9.

Descritores

Diabete melito; educação médica; vivência

\begin{abstract}
This study evaluated the effectiveness of theoretical and practical teaching method in diabetes and doctors' position about feasibility of intensive blood glucose control. Forty-eight internal medicine or endocrinology residents participated in a two-day diabetes immersion course. The participants received training on self-blood glucose monitoring, techniques of insulin administration and carbohydrate counting. They were also instructed to behave as patients with diabetes and to follow individual medical prescription. They were assessed through questionnaires. In knowledge assessment, a significant increase of $12 \%$ was observed between the beginning and the end of the course $(61.2 \%$ and $73.2 \%$, respectively, with $p<0.0001)$. Before the course, $70.8 \%$ and $89.6 \%$ of the participants believed there were complications in performing carbohydrate counting and blood glucose monitoring, respectively. After the experience, $82.9 \%$ of them had difficulties in carbohydrate counting and $80.8 \%$ in self-monitoring; $40.4 \%$ took all medications prescribed and $36.1 \%$ monitored blood glucose correctly. These results show that the methodology of this course is an effective way to disseminate knowledge and that it contributes to doctors becoming more sensitive to daily problems faced by patients with diabetes melito concerning the acceptance of medical recommendations. Arq Bras Endocrinol Metab. 2009;53(3):355-9.
\end{abstract}

Serviço de Diabetes,

Disciplina de Endocrinologia e Metabologia, Hospital das Clínicas, Faculdade de Medicina da Universidade de São Paulo (HC-FMUSP), São Paulo, SP, Brasil
Correspondência para:

Maria Cândida Ribeiro Parisi Secretaria de Endocrinologia Av. Dr. Enéas de Carvalho Aguiar, 255, $7^{\circ}$ andar - Cerqueira César 05403-000 - São Paulo, SP, Brasil emaildacandida@uol.com.br

Recebido em 25/Fev/2008 Aceito em 12/Jan/2009 


\section{INTRODUÇÃO}

$\mathrm{O}$

diabetes melito (DM) é uma doença crônica associada a várias complicações graves, sendo a principal causa de insuficiência renal, amputação não traumática de membros inferiores e perda visual em adultos $(1,2)$. Estudos prospectivos demonstraram que o arsenal terapêutico hoje disponível torna possível o controle do diabetes, reduzindo o risco de complicações (3-5); entretanto, muitas vezes a equipe médica falha em transformar as informações dos consensos em ferramentas úteis na prática clínica (6-8).

Os programas de educação médica continuada foram desenvolvidos para difundir novas informações entre os provedores de saúde e são mecanismos com potencial para diminuir as barreiras entre a prática clínica ideal e aquela em uso, bem como para aumentar o conhecimento técnico dos participantes (9).

Torna-se fundamental a compreensão de que apenas o conhecimento das alternativas terapêuticas não é suficiente para o adequado acompanhamento do portador de diabetes, pois muitas vezes o fator psicológico é o principal obstáculo para a intensificação no tratamento (10). Assim, é importante que os médicos tenham consciência das eventuais dificuldades enfrentadas pelos pacientes na realização do controle glicêmico intensivo, pois a vivência dessas dificuldades permite uma avaliação melhor do impacto gerado nos pacientes.

O presente estudo descreve os resultados do curso de imersão em diabetes, oferecido pelo Núcleo de Excelência no Atendimento ao Diabético (Nead) da Disciplina de Endocrinologia e Metabologia do Hospital das Clínicas da Faculdade de Medicina da Universidade de São Paulo (FMUSP). O curso foi idealizado com a finalidade de capacitar médicos clínicos a prestarem assistência ao portador de DM, proporcionando, simultaneamente, treinamento técnico e científico, bem como uma abordagem mais humanizada da doença.

\section{OBJETIVO}

Avaliar a eficácia do método de ensino teórico e prático em diabetes e a atitude de profissionais médicos quanto à realização de controle glicêmico intensivo.

\section{MÉTODOS}

Realizou-se em Pouso Alegre (MG) um curso intensivo de imersão em diabetes durante dois dias consecutivos voltado a médicos-residentes de clínica médica e endo- crinologia, cuja inscrição foi voluntária e gratuita. Os participantes foram conduzidos por um ônibus a um hotel, onde ficaram hospedados durante o curso.

O programa foi elaborado para a aquisição de conhecimento teórico e prático. No braço teórico, foram ministradas aulas expositivas por médicos-assistentes do Serviço de Diabetes da Disciplina de Endocrinologia e Metabologia, abordando-se temas como dificuldades no diagnóstico etiológico de diabetes, insulinoterapia, uso apropriado de antidiabéticos orais e prevenção de complicações crônicas. Conduziram-se, ainda, discussões de casos clínicos escolhidos de forma a permitir uma análise crítica das várias formas de insulinoterapia, desde bomba de infusão contínua, insulinização basalbolus com análogos de insulina e combinação de insulina basal ou prandial com antidiabéticos orais.

No braço prático, os participantes foram orientados a agir como portadores de DM tipo 1 em terapia intensiva, realizando-se automonitorização da glicemia capilar pré e pós-prandial, contagem de carboidratos nas refeições, simulação de aplicação de insulina (com soro fisiológico) e uso de medicamentos orais (placebo), conforme prescrição médica individualizada. Cada participante recebeu um monitor de glicemia capilar, um manual de contagem de carboidratos, seringas e canetas de insulina. Foram realizadas oficinas pedagógicas, nas quais os participantes foram divididos em quatro grupos, recebendo orientações quanto à aplicação de insulina, técnicas de rodízio, uso dos monitores de glicemia capilar, cuidados com os pés e avaliação de "pé em risco".

A avaliação da eficácia do método pedagógico foi realizada através de questionário, elaborado pela comissão organizadora do curso, constituído por 24 testes de múltipla escolha referentes a temas teóricos e práticos. Das 12 questões práticas, cinco foram designadas para avaliar conhecimentos em contagem de carboidratos e correção de glicemias pré-prandiais alteradas e cálculo do bolus prandial de insulina, enquanto outras cinco questões referiam-se a ajustes de horários e doses de insulina baseados em monitoração glicêmica domiciliar. Esse questionário foi aplicado aos profissionais médicos antes e imediatamente após o curso. A atitude dos participantes quanto ao controle glicêmico intensivo foi avaliada através de outro questionário, que abordava tanto a aplicabilidade da automonitorização glicêmica, contagem de carboidratos e múltiplas aplicações de insulina, como a aderência ao regime terapêutico prescrito durante o período do curso. Cada questão tinha 
pontuação de 1 a 5 , de acordo com o grau de assentimento com as afirmações.

\section{Análise estatística}

As comparações entre os resultados das avaliações pré e pós-curso foram feitas utilizando-se os testes ANOVA seguido por Bonferroni, D’Agostino e Pearson, através do programa GraphPad Prism versão 4.0.

\section{RESULTADOS}

Participaram do curso 48 médicos residentes, dos quais 19 eram residentes de clínica médica e 29 , de endocrinologia.

$\mathrm{Na}$ avaliação dos resultados, observamos um aumento significante de $12 \%$ no índice de acerto no questionário entre o início e o final do curso, que foi $61,2 \%$ e $73,2 \%$, respectivamente $(\mathrm{p}<0,0001)$ (Figura 1 ).

$\mathrm{O}$ resultado da avaliação particularizada do questionário encontra-se na tabela $\mathrm{l}$. $\mathrm{O}$ índice de acerto nas questões teóricas pré e pós-curso foram, respectivamente, $68,1 \%$ e $77,9 \%(\mathrm{p}<0,001)$ no grupo dos médicos-residentes de endocrinologia e 50,4\% e 64\% ( $\mathrm{p}<0,0372)$ no grupo de médicos-residentes de clínica médica.

Para as questões práticas que avaliaram conhecimentos para cálculo do bolus de insulina pré-prandial (contagem de carboidrato e correção de glicemia capilar alterada), a taxa de acerto antes do curso foi de $73,8 \%$ e $57,9 \%$ no grupo de residentes de endocrinologia e clínica médica, respectivamente. A avaliação final mostrou um aumento estatisticamente significativo nos dois grupos com $86,2 \%$ de acertos para residentes de endocrinologia $(\mathrm{p}=0,0340)$ e $83,1 \%$ para residentes de clínica médica $(\mathrm{p}<0,001)$. As questões destinadas à avaliação, à interpretação da monitoração e a consequente ajuste nas doses diárias de insulina foram as que se mostraram de maior dificuldade para os participantes, com menor percentagem de acertos tanto pré como pós-curso. No entanto, observou-se diferença estatisticamente significativa na avaliação pré e pós-curso nos dois grupos (Tabela 1 ).

A avaliação, realizada antes do curso, quanto à postura dos profissionais sobre a aplicabilidade do controle glicêmico intensivo, mostrou que $70,8 \%$ dos participantes acreditavam haver dificuldades para a realização da contagem de carboidratos e $89,6 \%$, para a realização de automonitorização glicêmica (Figuras 2 e 3 ).

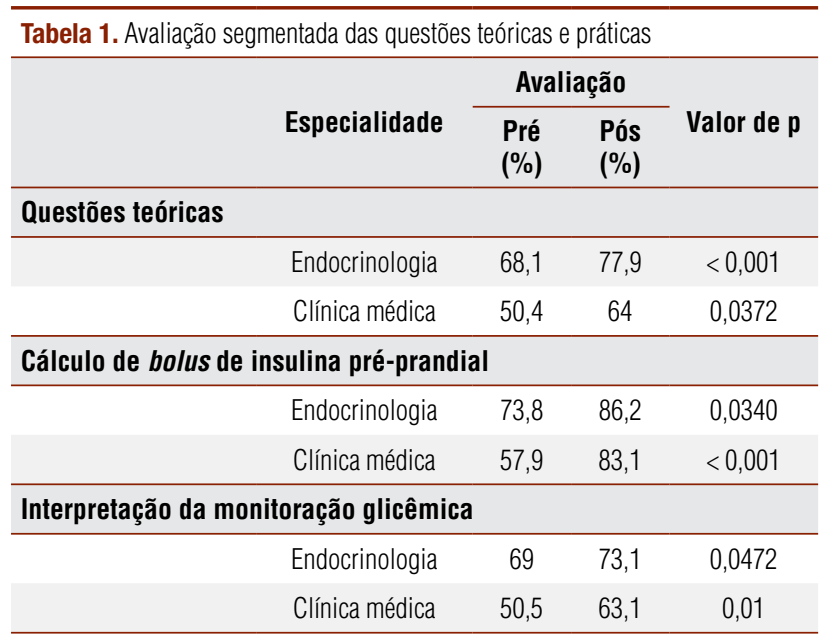

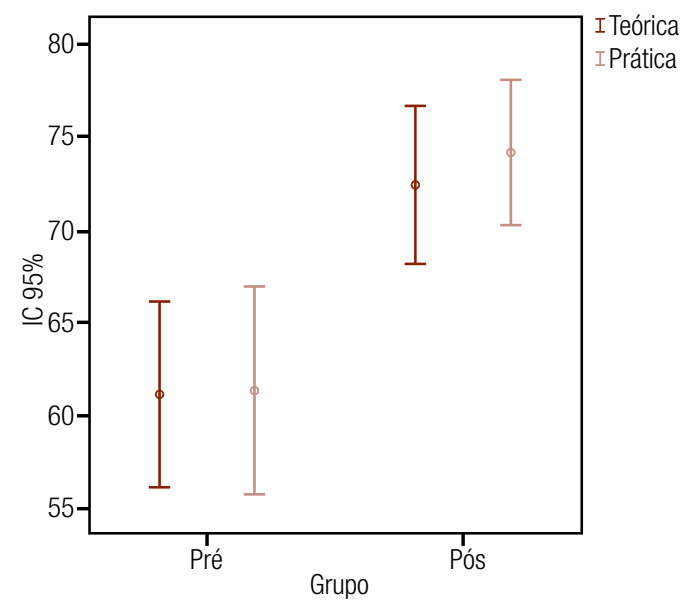

Figura 1. Índice de acertos pré e pós-curso de imersão em diabetes.

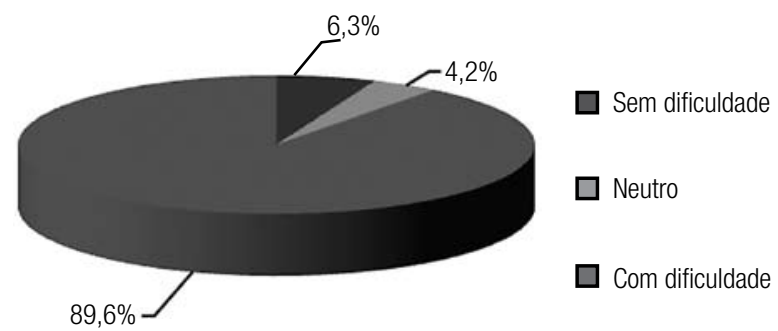

Figura 2. Percentual de participantes com dificuldades de realizar a automonitoração glicêmica.

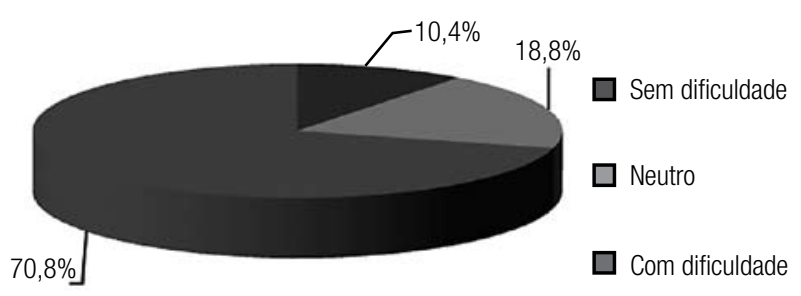

Figura 3. Percentual de participantes com dificuldades na contagem de carboidratos. 
Após a experiência prática, $82,9 \%$ dos participantes encontraram dificuldades na realização de contagem de carboidratos e $80,8 \%$, na automonitorização. Considerando a aderência ao tratamento, $40,4 \%$ dos médicos fizeram uso de todas as medicações prescritas e $36,1 \%$ monitorizaram todas as glicemias.

\section{DISCUSSÃO}

A importância do adequado manejo do DM e a rápida evolução das opções terapêuticas disponíveis tornam essencial a busca de alternativas pedagógicas que possam contribuir, de forma construtiva e concreta, no processo de aprendizado dos profissionais médicos. Não é possível listar de forma objetiva todos os aspectos envolvidos na abordagem terapêutica do DM. A complexidade da doença, assim como o avanço no entendimento de sua fisiopatologia e terapêutica, exige do profissional uma imensa gama de conhecimentos teóricos e práticos que possibilitem a obtenção de resultados satisfatórios no tratamento dos pacientes tanto na prevenção de complicações crônicas, como na melhoria da qualidade de vida.

Os resultados obtidos na avaliação teórica, pré e pós-curso de imersão, mostram importante aquisição de conhecimentos tanto no grupo de residentes de endocrinologia $(68,1 \%$ versus $77,9 \%)$, quanto de clínica médica ( $50,4 \%$ versus $64 \%)$. No entanto, o maior aprendizado promovido por essa forma de ensino foi a aquisição de conhecimentos práticos, principalmente no quesito contagem de carboidratos e correção da dose de insulina conforme controle de glicemia capilar, com índice final de acerto superior a $80 \%$ nos dois grupos, sendo o incremento obtido pelos residentes de clínica médica de $25,2 \%$, o que comprova a efetividade desse tipo de abordagem na disseminação de conhecimento $(9,11)$.

Vários estudos (12-14) sugerem que uma grande proporção de pacientes com diabetes tem dificuldade em gerenciar suas medicações (hipoglicemiantes orais e insulina) e em realizar autocuidados. Resultados obtidos pelo estudo DAWN (Diabetes Attitudes, Wishes and Needs) mostraram aderência ao regime terapêutico relatada pelos pacientes em $46 \%$ dos diabéticos tipo 1 e $39 \%$ dos portadores de tipo 2 , e foi maior para medicação, automonitorização e frequência às consultas do que para mudanças comportamentais (dieta e atividade física) (15). Por meio da metodologia aplicada, evidenciamos que, antes do curso, os profissionais envolvidos consideravam baixa a aplicabilidade da contagem de carboidratos e automonitoração glicêmica. Com a experiência prática de comportarem-se como diabéticos insulino-dependentes, $82,9 \%$ desses profissionais relataram dificuldades na realização da contagem de carboidratos, $80,8 \%$ na automonitoração e apenas $40,4 \%$ deles tiveram boa aderência ao tratamento proposto, deixando evidente que apenas um bom conhecimento teórico não é suficiente para o adequado manejo da doença. Em várias situações, nem mesmo o profissional entende e domina as práticas que delega ao paciente, supondo que estas não demandem treinamento e aprendizado ou que sejam habilidades de fácil aquisição e reprodutibilidade. Os programas de atualização médica continuada contribuem para o aumento de informações e atitudes favoráveis dos profissionais de saúde em relação ao diabetes $(9,11)$.

Os resultados deste estudo mostram que o tratamento do portador de diabetes exige da equipe profissional envolvida uma autoavaliação e atitudes mais positivas, no intuito de minimizar a lacuna entre o controle glicêmico intensivo e formas de atingi-lo. A colocação de Caprara e Franco (16) de que, no processo terapêutico, além do suporte técnico-científico, "conhecer a realidade do paciente, ouvir suas queixas e encontrar estratégias que facilitem sua adaptação ao estilo de vida exigido pela doença" é uma peça-chave e, ao mesmo tempo, sintetiza o objetivo do presente projeto: realizar um curso de imersão em diabetes como técnica educativa para profissionais médicos.

Agradecimentos: Karla F. S. Melo, Maria E. E. R. Silva, Maria L. C. Gianella.

Declaração: os autores declaram não haver conflitos de interesse científico neste artigo.

\section{REFERÊNCIAS}

1. Amos AF, McCarty DJ, Zimmet P. The rising global burden of diabetes and its complications: estimates and projections to the year 2010. Diabet Med. 1997;14 Suppl 5:1-85.

2. Chaturvedi N.The burden of diabetes and its complication: trends and implications for intervention. Diabetes Res Clin Pract. 2007;76 Suppl 1:S3-12

3. The Diabetes Control and Complications Trial Research Group. The effect of intensive treatment of diabetes on the development and progression of long-term complications in insulin-dependent diabetes mellitus. N Engl J Med. 1993;329(14):977-86.

4. No authors listed. Intensive blood-glucose control with sulphonylureas or insulin compared with conventional treatment and risk of complications in patients with type 2 diabetes (UKPDS 33). UK Prospective Diabetes Study (UKPDS) Group. Lancet. 1998;352(9131):837-53. 
5. No authors listed. Effect of intensive blood-glucose control with metformin on complications in overweight patients with type 2 diabetes (UKPDS 34). UK Prospective Diabetes Study (UKPDS) Group. Lancet. 1998;352(9131):854-65.

6. Fain JA, Melkus GD. Nurse practitioner practice patterns based on standards of medical care for patients with diabetes. Diabetes Care. 1994;17(8):879-81.

7. Weiner JP, Parente ST, Garnick DW, Fowles J, Lawthers AG, Palmer $\mathrm{RH}$. Variation in office-based profile of care provided to Medicare patients with diabetes. JAMA. 1995;273(19):1503-8.

8. Tuttleman M, Lipsett L, Harris MI. Attitudes and Behaviors of primary care physicians regarding tight control of blood glucose in IDDM patients. Diabetes Care. 1993;16(5):765-72.

9. Davis DA, Thomson MA, Oxman AD, Haynes RB. Evidence for the effectiveness of $\mathrm{CME}$. A review of 50 randomized controlled trials. JAMA. 1992;268(9):1111-7.

10. Simmons D, Lillis S, Swan J, Haar J. Discordance in perceptions of barriers to diabetes care between patients and primary care and secondary care. Diabetes Care. 2007;30(3):490-95.
11. Sharp LK, Lipsky MS. The short-term impact of a continuing medical education program on providers' attitude toward treating diabetes. Diabetes Care. 1999;22(12):1929-32.

12. Pugh MJ, Anderson J, Pogach LM, Berlowitz DR. Differential adoption of pharmacotherapy recommendations for type 2 diabetes by generalists and specialists. Med Care Res Rev. 2003;60(2):178-200.

13. Johnson SB. Methodological issues in diabetes research. Measuring adherence. Diabetes Care. 1992;15(11):1658-67.

14. Morris AD, Boyle DI, McMahon AD, Greene SA, MacDonald TM, Newton RW. Adherence to insulin treatment, glycaemic control, and ketoacidosis in insulin-dependent diabetes mellitus. The DARTS/ MEMO Collaboration. Diabetes Audit and Research in Tayside Scotland. Medicines Monotoring Unit. Lancet. 1997;350(9090):1505-10.

15. Peyrot M, Rubin RR, LauritzenT, Snoek FJ, Matthews DR, Skovlund SE. Psychosocial problems and barriers to improved diabetes management: results of the Cross-National Diabetes Attitudes, Wishes and Needs (DAWN) Study. Diabet Med. 2005;22(10):1379-85.

16. Caprara A, Franco ALS. A relação paciente-médico: para uma humanização da prática médica. Cad Saúde Pública 1999;15(3):647-54. 